\title{
Certificación de la Mantenibilidad del Producto Software: Un Caso Práctico
}

\author{
Moisés Rodríguez \\ Alarcos Quality Center, Universidad de \\ Castilla-La Mancha \\ Ciudad Real, España \\ moises.rodriguez@alarcosqualitycenter.com
}

\author{
Óscar Pedreira \\ Laboratorio de Base de Datos, \\ Universidad de la Coruña \\ A Coruña, España \\ oscar.pedreira@udc.es
}

\author{
Carlos Manuel Fernández \\ AENOR, Asociación Española de \\ Normalización y Certificación \\ Madrid, España \\ cmfernandez@aenor.es
}

\begin{abstract}
Resumen - La calidad del software está adquiriendo durante los últimos años una gran importancia, principalmente debido a que el software está presente en prácticamente todo lo que nos rodea $y$ se hace necesario asegurar su correcto funcionamiento. Sin embargo, hasta ahora la mayor parte de los estudios se han centrado en evaluar la calidad de los procesos de desarrollo, y aunque existen trabajos centrados en la calidad del producto software, no existe todavía una propuesta completa para la evaluación y certificación de la calidad del producto software, basada en la nueva familia de normas ISO/IEC 25000. El presente artículo expone un caso de estudio de evaluación, mejora y certificación de la mantenibilidad de un producto software, en el que han participado una empresa desarrolladora de software, un laboratorio de evaluación acreditado y una entidad de certificación. Para ello, se presentarán las características de este tipo de evaluaciones, el rol que cumple cada una de las entidades participantes y el proceso seguido desde la evaluación inicial hasta la certificación definitiva.
\end{abstract}

Palabras Clave - ISO/IEC 25000, calidad producto software, certificación, laboratorio acreditado, mantenibilidad, modelo de calidad.

\section{INTRODUCCIÓN}

Hoy en día el software está en prácticamente todo lo que nos rodea, no solo en ordenadores, móviles o tablets, sino en los electrodomésticos, en los vehículos, en el sector de la medicina, en la banca y seguros, etc. Este aumento en la demanda de productos software ha dado lugar a un crecimiento de las empresas y departamentos encargados de su desarrollo, lo que se conocen como "software factories" [1]. Por otro lado, la falta de personal especializado para ciertas tareas del desarrollo software, así como la búsqueda de la reducción de costes han dado lugar a lo que se conoce como "outsourcing" del desarrollo software, de manera que las empresas externalizan todo o parte de las actividades de desarrollo software a otros departamentos o empresas. Sin embargo, cuando se externaliza aumentan los riesgos y la falta de control sobre la calidad del software que la empresa contratada entrega, surgiendo la necesidad de evaluar y asegurar la calidad del software de dichas empresas.

Aunque desde hace bastantes años la evaluación de la calidad del software es un campo de gran actividad tanto investigadora como en el sector industrial, la mayor parte del esfuerzo realizado se ha centrado en la calidad de los procesos, habiéndose desarrollado gran cantidad de modelos y estándares de referencia, evaluación y mejora de procesos software: ISO 90003, ISO 12207, ISO 15504, CMM, CMMI, IDEAL, SCAMPI, etc., en los que numerosas empresas de todo el mundo se han evaluado y/o certificado. Sin embargo, es cada día mayor el número de organizaciones y empresas que se interesan, no solo por la calidad de los procesos que se siguen en el desarrollo de software, sino también por la calidad de los productos que desarrollan y/o adquieren, ya que una vez que el producto ha sido implantado en sus instalaciones se encuentran con graves problemas de calidad y complicaciones a la hora de corregirlo, adaptarlo o evolucionarlo [2].

Desde hace varias décadas se han elaborado también trabajos de investigación, normas y estándares, con el objetivo de crear modelos, procesos y herramientas de evaluación de la calidad del propio producto software. Entre los más representativos podemos citar: [3-6] y la nueva familia de normas ISO/IEC 25000 [7]. Sin embargo, la certificación de la calidad del producto software sigue siendo a día de hoy un área relativamente joven de la Ingeniería del Software, en la que todavía no existe un consenso definitivo.

Por este motivo y con el objetivo de conocer los trabajos existentes sobre certificación de la calidad del producto software, en 2012 se realizó la revisión sistemática expuesta en [8] siguiendo la guía propuesta por Kitchenham en [9], junto con la plantilla descrita por Biolchini en [10]. Como resultado se obtuvo un conjunto de 10 estudios primarios que cumplían con los requisitos de búsqueda, reflejados en la Tabla 1 .

TABLA I. Listado DE ESTUdios Primarios SELECCIONADOS

\begin{tabular}{|c|c|c|c|}
\hline Id & Título & Año & Referencia \\
\hline 1 & A software product certification model & 2010 & {$[11]$} \\
\hline 2 & $\begin{array}{c}\text { Embedded software component quality and } \\
\text { certification }\end{array}$ & 2009 & {$[12]$} \\
\hline 3 & $\begin{array}{c}\text { Performance certification of software } \\
\text { components }\end{array}$ & 2011 & {$[13]$} \\
\hline 4 & $\begin{array}{c}\text { Requirements certification for offshoring } \\
\text { using LSPCM }\end{array}$ & 2010 & {$[14]$} \\
\hline 5 & $\begin{array}{c}\text { SCfM_PROD: A software product } \\
\text { certification model }\end{array}$ & 2008 & {$[15]$} \\
\hline 6 & $\begin{array}{c}\text { Continuously ensuring quality through } \\
\text { software product certification: A case study }\end{array}$ & 2010 & {$[16]$} \\
\hline 7 & $\begin{array}{c}\text { A Software Certification Consortium and its } \\
\text { Top 9 Hurdles }\end{array}$ & 2009 & {$[17]$} \\
\hline 8 & Software component certification & 2001 & {$[18]$} \\
\hline 9 & $\begin{array}{c}\text { Standardized code quality benchmarking for } \\
\text { improving software maintainability }\end{array}$ & 2011 & {$[19]$} \\
\hline 10 & $\begin{array}{c}\text { Towards a software component certification } \\
\text { framework }\end{array}$ & 2007 & {$[20]$} \\
\hline
\end{tabular}

Entre las principales conclusiones obtenidas se pueden destacar las siguientes:

- La mayoría de los estudios destacan el trabajo que ya existe en la certificación de procesos de desarrollo software y la necesidad de que esta certificación sea también extendida a las características del producto. 
- La mayoría de los estudios se basan en características de calidad extraídas de normas internacionales como la ISO/IEC 9126, utilizando métodos de evaluación también estándares como los de la ISO/IEC 14598. Sin embargo, ninguna de las propuestas ha adoptado el nuevo modelo y proceso de la familia ISO/IEC 25000.

- Aunque ya existen varias propuestas para certificar el producto software, la mayoría de ellas carece o tiene un número reducido de aplicaciones reales en la industria del software.

- La mayoría de los estudios utiliza indistintamente los conceptos de evaluación y certificación, lo que se considera un error. Es necesario diferenciar entre el proceso de evaluación, realizado frente a un modelo de calidad, y el posterior proceso de certificación realizado por un organismo acreditado e independiente.

- Un denominador común de los estudios que presentan casos de aplicación práctica es la importancia que atribuyen a disponer de una herramienta que automatice las actividades.

Por todo lo anterior, el objetivo del presente artículo es presentar las características principales de una propuesta completa para la evaluación y certificación del producto software basadas en la familia ISO/IEC 25000 y demostrar su aplicación mediante un caso de estudio práctico. Para ello, el resto del artículo se estructura de la siguiente manera: en el apartado 2 se presenta el modelo de AENOR (Asociación Española de Normalización y Certificación) para Gobierno de las TICs basado en estándares ISO, dentro del cual se ha incluido la nueva familia de normas ISO/IEC 25000. En el apartado 3 se resumen los principales elementos de la evaluación, haciendo especial hincapié en el ecosistema de evaluación y en el laboratorio acreditado AQC Lab. En el apartado 4 se presenta el flujo de trabajo que se sigue para la certificación del producto una vez ha sido evaluado por un laboratorio acreditado, así como los resultados de un caso práctico de evaluación, mejora y certificación de un producto software. Y finalmente, el apartado 5 presenta las conclusiones obtenidas con este trabajo y las líneas futuras de investigación.

\section{MODELO DE AENOR PARA TICS CON ESTÁNDARES ISO}

Desde al año 2006, AENOR ha presentado y desarrollado un modelo de Gobierno y Gestión de las TIC para el siglo XXI (Fig. 1) basado en normas ISO [21] con las mejores prácticas del sector. Este modelo ha tenido una gran aceptación en el mundo empresarial público y privado, debido a que está orientado a la mejora de la productividad, la innovación y el ahorro de costes; con una especial directriz que cumple con los objetivos de las organizaciones.

Básicamente, el modelo propone dos grandes áreas y tres certificaciones. El área de producción diaria de un Centro de Procesamiento de Datos (CPD), con los objetivos de calidad y seguridad de los servicios de TI (ISO/IEC 20000-1 e ISO 27001); y el área de ingeniería o desarrollo del software con calidad y madurez en sus procesos con SPICE ISO 15504 -ISO 12207. Como características de este modelo hay destacar las siguientes:

- Es un modelo avalado por más de 150 países que han participado en el desarrollo e implantación de los estándares ISO que en él se recogen. Esto demuestra la madurez e importancia que el modelo y las normas en las que se apoya tienen dentro del sector de las TICs.

- Es un modelo dinámico, puesto que se encuentra en constante evolución, tanto por los avances que van saliendo en la industria de las TICs y que son incluidos en el mismo, como por las nuevas versiones de los estándares que lo forman que igualmente son adaptadas y consideradas.

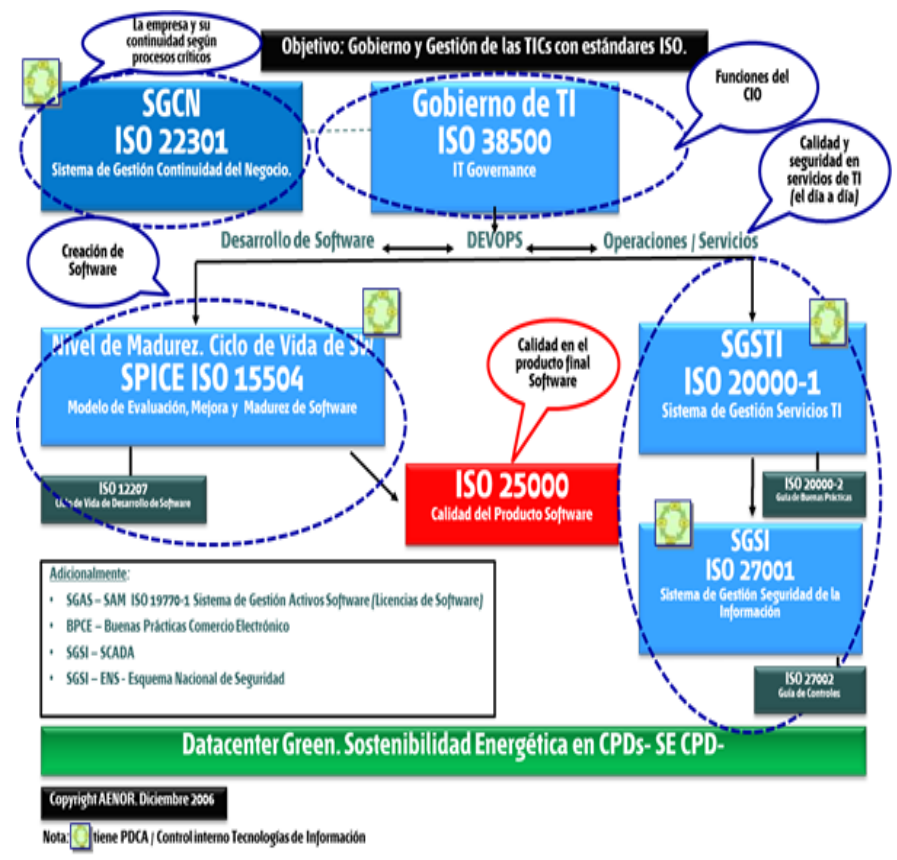

Fig. 1. Modelo de AENOR para Gobierno y Gestión de las TICs con estándares ISO [22]

Recientemente y después de un período de maduración y consolidación del modelo, en especial en el área de desarrollo de software o factorías de software, se ha considerado oportuno asumir una nueva certificación según la Norma ISO/IEC 25000 de producto software. En un principio centrada en una de las características, la mantenibilidad, para continuar próximamente con otras características de la propia ISO/IEC 25000.

De manera básica pero ilustrativa, lo que se pretende con este nuevo certificado de producto software es algo equivalente a lo que representa el sello EuroNCAP (European New Car Assessment Programme) en la industria del automóvil. Es decir, igual que cuando compramos un vehículo queremos que haya sido revisado y tenga las máximas estrellas EuroNCAP, este nuevo certificado permite conocer la calidad de los productos software en base a una norma internacional como es la ISO/IEC 25000 y poder optar por la opción que mejor se adecua a nuestras necesidades

\section{EVALUACIÓN DE LA CALIDAD DEL PRODUCTO}

Una vez presentado el modelo de AENOR para gobierno de las TICs basado en normas ISO, en el presente apartado se detallarán los elementos necesarios para llevar a cabo la evaluación de la calidad del producto software.

\section{A. Familia de normas ISO/IEC 25000}

La familia de normas ISO/IEC 25000 tiene por objetivo la creación de un marco de trabajo común para evaluar la calidad del producto software, sustituyendo a las anteriores ISO/IEC 9126 e ISO/IEC 14598 y convirtiéndose así en la piedra angular de esta área de la Ingeniería del Software. La familia ISO/IEC 25000 se encuentra compuesta de varias partes o divisiones, entre las que podemos destacar:

- La norma ISO/IEC 25010 [23] que define varios modelos de calidad y determina las características de calidad del producto software que se pueden evaluar (Fig. 2). En total son 8 las características de calidad que identifica: 


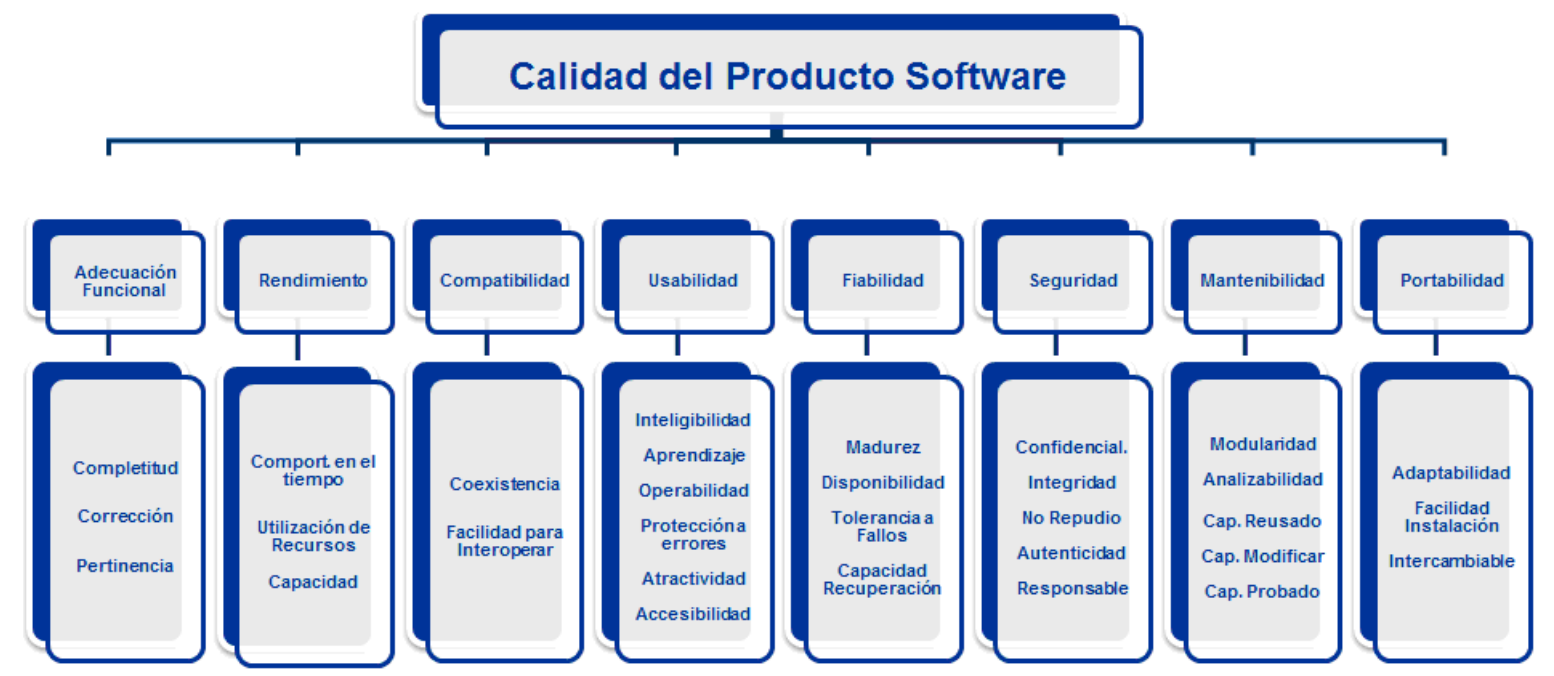

Fig. 2. Modelo de calidad del producto software según la ISO/IEC 25010

funcionalidad, rendimiento, compatibilidad, usabilidad, fiabilidad, seguridad, mantenibilidad y portabilidad. Esta parte de la norma representa por tanto el espejo frente al cual podemos mirar la calidad de nuestro producto software.

- La norma ISO/IEC 25040 [24] que define el proceso de evaluación, compuesto por cinco actividades, que determinan las tareas a realizar para poder evaluar la calidad del producto software: Establecer los requisitos, Especificar la evaluación, Diseñar la evaluación, Ejecutar la evaluación y Concluir la evaluación.

- La norma ISO/IEC 25020 que será la encargada de definir las métricas de calidad del producto software y que todavía está en pendiente de publicación.

\section{B. Requisitos para la evaluación y certificación del producto}

A partir de todo lo anterior, se pueden extraer los requisitos principales para poder llevar a cabo la evaluación y certificación de la calidad del producto software, que además de personal experimentado, se pueden concretar en los siguientes tres elementos: un modelo de calidad, un proceso de evaluación y un entorno tecnológico que de soporte a los dos elementos anteriores.

\section{1) Modelo de Calidad}

El objetivo del modelo es definir las características del producto software que se pue-den evaluar y que por tanto influyen en la calidad del mismo, es decir, el modelo deja claro los puntos de vista desde los que se puede considerar la calidad de un producto software. Es importante remarcar que el modelo define el qué evaluar (características de calidad), pero no el cómo evaluarlas. Aspectos como la funcionalidad que tiene un producto, la usabilidad, la seguridad o la mantenibilidad del producto software podrían ser algunos de estos tipos de características.

Es importante que el modelo no solo defina las características de calidad, sino que también desglose éstas en subcaracterísticas de más bajo nivel y a su vez estas en indicadores y métricas que se puedan medir a partir de los elementos del producto software. El modelo también debe identificar, si es que existen, las relaciones entre las características de calidad del producto. De manera que podamos saber si mejorar una característica supone mejorar a su vez otra, o si por el contrario una mejora en una característica supone una pérdida en otra de dichas características.

Moisés Rodríguez, Óscar Pedreira y Carlos M. Fernández. 2015. Certificación de la Mantenibilidad del Producto Software: Un Caso Práctico Revista Latinoamericana de Ingeniería de Software, 3(3): 127-134, ISSN 2314-2642
Y probablemente la parte más complicada a la hora de definir el modelo de calidad, es disponer de unos umbrales contrastados y validados que reflejen el valor de calidad que se quiere alcanzar, es decir, por ejemplo cómo saber si cuando decimos que un producto tiene un $4 \%$ de código duplicado, eso es bueno o malo y cómo de bueno o malo es.

Como se ha comentado anteriormente, la nueva familia de normas ISO/IEC 25000 contempla un modelo para la calidad del producto software que define las características y subcaracterísticas de calidad que se pueden medir. La dificultad es que esta familia de normas no determina un conjunto concreto de indicadores, métricas y umbrales que puedan ser tomados por las organizaciones como referente a la hora de evaluar y poder certificar la calidad de sus productos.

\section{2) Proceso de Evaluación}

El objetivo del proceso de evaluación es definir las actividades que se deben realizar para poder llevar a cabo la evaluación de la calidad del producto software. Es importante remarcar que el proceso de evaluación define el cómo evaluar, pero no el qué evaluar, que como veíamos antes estaba definido por el modelo.

El proceso de evaluación suele iniciarse por la selección de las partes del producto software que se van a evaluar: el código fuente, los requisitos, los modelos, los casos de pruebas, el producto en ejecución, etc. podrían ser algunos de los ejemplos de partes del producto software que se pueden evaluar. Además, durante el proceso se debe también identificar qué características se van a evaluar. Características de calidad que se han definido previamente en un modelo de calidad como hemos visto en el apartado anterior, de ahí la relación entre el proceso de evaluación y el modelo de calidad.

El proceso de evaluación debe definir el conjunto de pasos que el evaluador debe seguir, identificar las herramientas que se utilizarán, así como identificar qué personas participarán en la evaluación y cuáles serán las actividades en las que participarán.

El objetivo último del proceso de evaluación es generar un informe con los resulta-dos obtenidos, asegurando que dicho informe sea completo, repetible y legible para el público objetivo del mismo.

La ISO/IEC 25040:2011 también define un proceso de evaluación muy completo, cuyas actividades se han enumerado en la introducción del presente artículo. Aquí la dificultad es 
que este proceso no está preparado para la certificación del producto software, sino solo para la parte de evaluación. Por tanto es necesario complementarlo con otro conjunto de pasos.

\section{3) Entorno tecnológico de soporte}

El objetivo del entorno tecnológico es precisamente el de asistir al proceso de evaluación y a la recolección de las métricas y umbrales definidos en el modelo de calidad.

Las herramientas que forman este tipo de entornos sirven para facilitar la obtención de los datos, ya sea parseando automáticamente el producto software o permitiendo al evaluador introducir los datos de una manera amigable. Estas herramientas deben poder también automatizar los algoritmos de medición, de manera que a partir de las métricas base que se hayan tomado, se puedan ir escalando dichos valores para obtener los indicadores de calidad. Además, estas herramientas también deben permitir presentar los resultados de una manera entendible dependiendo el público objetivo de los mismos.

Dentro de este tipo de herramientas hay que diferenciar aquellas que realizan medición de la calidad del producto software, obteniendo métricas de bajo nivel e indicadores. De aquellas herramientas que evalúan la calidad del producto software, escalando las métricas anteriores para dar una valoración de las subcaracterísticas y características de calidad del modelo. De aquí la relación también entre el modelo y el entorno tecnológico.

Aunque hoy en día existen ya en el mercado muchas herramientas de este tipo [25], todavía no existe ninguna que esté alineada a un modelo de calidad que siga la familia ISO/IEC 25000, de ahí la dificultad para poder elegir una que se adapte a las necesidades de una organización que quiera certificar la calidad de sus productos.

Una vez detallados los requisitos necesarios para la evaluación y certificación del producto y vistas las dificultades de las propuestas existentes, en el siguiente apartado se presenta el ecosistema creado y las soluciones adoptadas.

\section{Ecosistema para la evaluación y certificación del producto software}

La certificación, entendida como la evaluación por una entidad independiente y acreditada para llevar a cabo auditorías de producto software, es un ámbito que hasta ahora no está contemplado por la familia de normas ISO/IEC 25000 y que sin embargo, es de gran interés para los desarrolladores y/o adquisidores de producto software, ya que les permitiría identificar rápidamente la calidad de un producto software de una manera estandarizada.

Por esta razón, AENOR (Asociación Española de Normalización y Certificación) y el laboratorio de evaluación de la calidad del producto software AQC Lab, han elaborado una certificación basada en la familia de normas ISO/IEC 25000. Dadas las dificultades vistas en el apartado anterior para evaluar la calidad del producto en base a la ISO/IEC 25000, junto con la novedad de esta nueva certificación y por tanto el desconocimiento del sector sobre las necesidades para su implantación, se ha empezado a construir lo que se ha denominado como Ecosistema para la Evaluación y Certificación de la Calidad del Producto Software, con el objetivo de resolver las dificultades expuestas e identificar todas las entidades que deben participar en el proceso que permita obtener un certificado para el producto software.

El ecosistema está formado inicialmente por las siguientes entidades (Fig. 3):

- Organizaciones que quieren certificar la calidad del producto. Estas organizaciones pueden ser tanto empresas desarrolladoras de software (de cualquier dominio), como entidades que han externalizado sus desarrollos o directamente adquieren un producto software, independientemente del propósito del mismo. Esta entidad es el elemento central del ecosistema y su interés por la calidad del producto es el que motiva precisamente la evaluación y certificación del mismo.

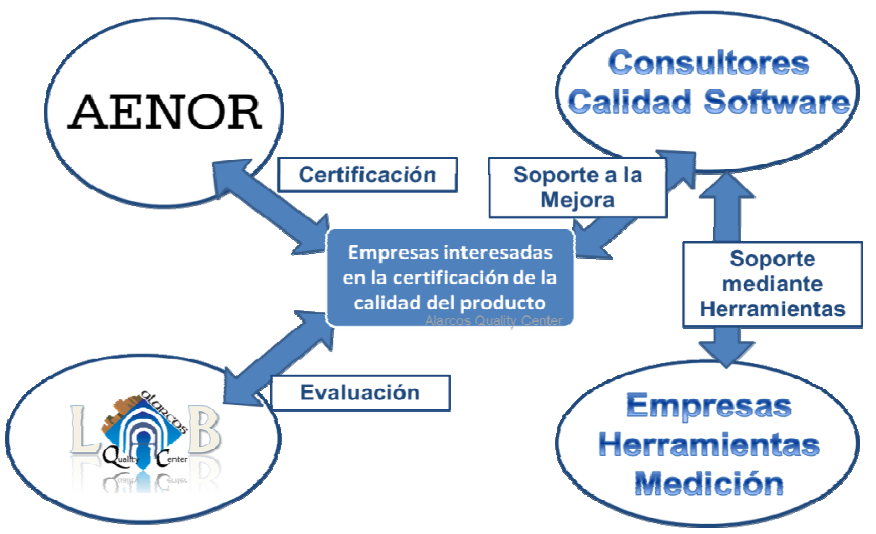

Fig. 3. Modelo de calidad del producto software según la ISO/IEC 25010

- Organismo certificador acreditado. En este caso dicho organismo es AENOR, quién tiene la experiencia de más de 20 años realizando auditorías de calidad de productos en otros sectores y que desde 2006 viene desarrollando el modelo para gobierno de las TIC basado en normas ISO [21], en el que recientemente ha incluido la propia norma ISO/IEC 25000 para llevar a cabo este tipo de auditorías de calidad del producto software. Para ello, AENOR ha desarrollado un reglamento interno de certificación del producto, de manera que a partir de un informe de evaluación emitido por un laboratorio acreditado y de una auditoría en las instalaciones de la empresa desarrolladora del producto, está en disposición de emitir un certificado sobre el nivel de calidad del mismo. Gracias a esta entidad del ecosistema, se complementa el proceso de evaluación, solucionando la dificultad que se identificaba anteriormente por la falta de un proceso de certificación.

- Laboratorio acreditado para la evaluación de la calidad del producto. En este caso dicha entidad del ecosistema es AQC Lab. Una de las necesidades que se identificaron inicialmente para poder evaluar la calidad del producto software, fue disponer de una entidad externa capaz de emitir una evaluación independiente sobre el producto software. Con esta idea, en 2009 comienza la construcción de AQC Lab, un laboratorio que basado en la familia ISO/IEC 25000 permita, tanto a empresas desarrolladoras de software como a entidades que externalizan o adquieren software, disponer de un informe independiente que refleje la calidad del producto software. Con el objetivo de obtener un reconocimiento a la validez de las evaluaciones realizadas por AQC Lab, se decidió elaborar toda la infraestructura de gestión necesaria para conseguir la acreditación, siguiendo además para ello prácticas del desarrollo ágil como se expone en [26]. El resultado fue que en 2012 AQC Lab conseguía la acreditación de ENAC (Entidad Nacional de Acreditación) en la norma ISO/IEC 17025 [27], como el primer laboratorio para la evaluación de la calidad de aplicaciones software bajo la familia de normas ISO/IEC 25000. La acreditación de acuerdo a la norma ISO/IEC 17025 confirma la competencia técnica del laboratorio y 
garantiza la fiabilidad en los resultados de los ensayos realizados.

AQC Lab cuenta con los tres requisitos principales identificados anteriormente y que se han detallado previamente en [28]:

- Un Proceso de Evaluación, que adopta directamente la norma ISO/IEC 25040:2011, y la completa con los roles concretos del laboratorio y los procedimientos de trabajo desarrollados.

- Un Modelo de Calidad, que define las características, métricas y umbrales para evaluar el producto software, complementando así la norma ISO/IEC 25010:2011.

- Un Entorno tecnológico propio, formado por varios niveles de herramientas que permiten automatizar en gran medida las tareas de medición y evaluación del producto.

Gracias a esta entidad del ecosistema, se ha solucionado la dificultad de contar con un modelo de calidad del producto detallado hasta las métricas y umbrales, así como de disponer de un laboratorio acreditado para la emisión de informes de evaluación sobre el producto software, que son la entrada al proceso de certificación.

- Consultores expertos en calidad de software. Antes de que las empresas puedan presentarse a una evaluación y certificación oficial, es necesario que cuenten con el apoyo y soporte a la mejora de sus productos ofrecido por consultores (internos o externos) que les permita controlar la calidad del producto a lo largo de todo el ciclo de vida $\mathrm{y}$ afrontar la certificación con garantías. Gracias a esta entidad del ecosistema, se soluciona la dificultad de contar con personal con experiencia para poder asegurar la calidad del producto software.

- Empresas desarrolladoras de herramientas para la medición del producto software. A su vez, tanto el laboratorio de evaluación como los consultores expertos en calidad, necesitan de soporte al proceso mediante herramientas de medición. Por ello, son necesarias empresas que desarrollen este tipo de herramientas y que alineen las mismas a las mediciones y umbrales que después serán objeto de evaluación y certificación. Entre este tipo de herramientas se puede destacar Kiuwan ${ }^{1}$, que ya ha comenzado a alinear sus mediciones al modelo de calidad del laboratorio AQC Lab, u otras de software libre como SonarQube ${ }^{2}$. Gracias a este tipo de entidades en el ecosistema, se solucionan las necesidades de contar con entornos tecnológicos que den soporte a la medición y la evaluación del producto.

De esta manera, por medio de estas herramientas, estos expertos en calidad software, del laboratorio AQC Lab y de AENOR, las empresas pueden evaluar, mejorar y certificar la calidad de sus productos software aplicando la familia ISO/IEC 25000, conformándose así el primer ecosistema completo para llevar a cabo este proceso.

\section{PILOTO DE EVALUACIÓN Y CERTIFICACIÓN DE LA CALIDAD DEL PRODUCTO SOFTWARE}

Una vez alcanzada la acreditación del laboratorio, se estableció un proceso de trabajo con AENOR que permitiera que los productos software, una vez se hubieran evaluado y obtenido un nivel adecuado de calidad, pudieran también conseguir un certificado.
Como resultado se creó un procedimiento que, a partir del informe del laboratorio acreditado y tras una auditoría por parte de AENOR, permitía certificar la calidad del producto software bajo estudio realizando los siguientes pasos (Fig. 4):

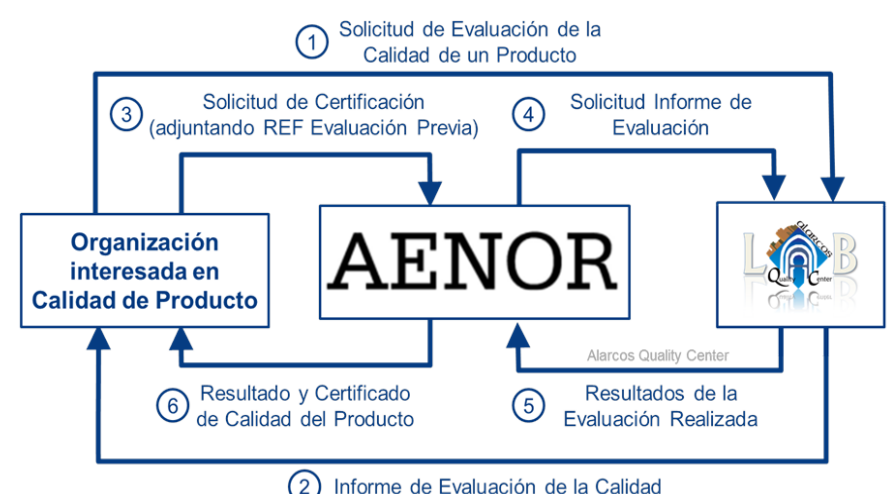

Fig. 4. Ciclo de Evaluación y Certificación del Producto Software

- Paso 1: El proceso comienza cuando la organización interesada en la calidad del producto software solicita una evaluación al laboratorio acreditado AQC Lab. Para ello debe rellenar un formulario con las características del producto software que se quiere evaluar, que es analizado por el laboratorio para emitir un contrato de evaluación con las condiciones del servicio. Aceptado este contrato, la organización hace entrega al laboratorio del producto software a evaluar. A partir de aquí, AQC Lab haciendo uso del entorno basado en ISO/IEC 25000 y acreditado por ENAC (modelo, proceso y herramientas) detallado en [28], realiza la evaluación. Este proceso suele tener una duración estimada de 2-3 semanas, dependiendo de las características del producto bajo evaluación.

- Paso 2: El resultado del paso anterior es un informe de evaluación con los resultados obtenidos, que es entregado a la organización solicitante. En este paso, puede ocurrir que el nivel de calidad obtenido por el producto software no sea suficientemente bueno, en cuyo caso la organización solicitante, apoyada por los consultores expertos del ecosistema, deberán refactorizar el producto para mejorar el nivel de calidad. En este caso, el tiempo que puede transcurrir dependerá el número de defectos que se deben solucionar y de la cantidad de recursos que la organización pueda dedicar para tal fin. Una vez refactorizado el producto, la organización deberá repetir el paso 1 del proceso para volver a obtener un informe de evaluación favorable.

- Paso 3: Cuando el producto software ha obtenido en la evaluación un nivel de calidad favorable, la organización podrá contactar con AENOR solicitando la certificación del producto e indicando la referencia previa de la evaluación que ha pasado realizada por un laboratorio acreditado.

- Pasos 4 y 5: AENOR contacta con AQC Lab (Paso 4) para comprobar que la empresa realmente ha evaluado su producto de software y el nivel obtenido es adecuado para la certificación. En este caso, el Laboratorio facilita a AENOR el informe de evaluación (paso 5), quien lo contrasta con la información facilitada por la empresa.

- Paso 6: Finalmente AENOR, analizará el informe de evaluación facilitado por el laboratorio y realizará una visita a la organización solicitante para, siguiendo con su reglamento interno de auditoría definido para el producto software, revisar el producto y las características del 
mismo. Como resultado de este proceso de auditoría de certificación, AENOR emitirá un informe y entregará a la organización un certificado que acredite la calidad del producto software evaluado. Este informe identifica entre otros a la organización solicitante, el producto certificado y su versión concreta, las características de calidad del modelo evaluadas y el informe del laboratorio acreditado que recoge los resultados de evaluación sobre los que se soporta el certificado emitido. Todo lo anterior permitió realizar a lo largo de 2013 un proyecto piloto de evaluación y certificación de los primeros productos software [22]. El listado actualizado de productos certificados se puede encontrar en la Web de iso $25000^{3}$. A continuación, en los siguientes apartados se presenta el proceso concreto seguido por una de las empresas que participó en dicho proyecto piloto, detallando la experiencia vivida y las mejoras realizadas a su producto software.

\section{A. Presentación de la empresa y del producto evaluado}

Enxenio S.L. se dedica al diseño, desarrollo y operación de sistemas de información para todo tipo de organizaciones. Creada en 2004, cuenta con una plantilla de unas 20 personas, la mayoría de ellas dedicadas a actividades de análisis, diseño y desarrollo de sistemas de información. Enxenio ha desarrollado varios productos software propios, y también ofrece a sus clientes servicios de desarrollo a medida. Sus principales áreas de trabajo son los sistemas de información geográfica (GIS), aplicaciones para la gestión de contenidos digitales para los sectores educativo y editorial, comercio electrónico y sistemas de gestión y soporte de procesos de negocio.

Los esfuerzos de gestión y mejora de la calidad que Enxenio ha llevado a cabo hasta este proyecto piloto se han centrado en la calidad de sus procesos de gestión de proyectos, desarrollo de software y seguridad de la información. Desde 2009 Enxenio cuenta con un sistema de gestión de seguridad de la información certificado bajo ISO 27001, y un sistema de gestión de proyectos y desarrollo de software certificado en ISO $12207 /$ ISO 15504 - nivel 2. Así, el proyecto piloto que se describe en esta sección supuso un cambio hacia la evaluación y certificación no solo de los procesos de la organización, sino también de los productos desarrollados.

El producto evaluado en el proyecto piloto de evaluación y certificación es $x$ Cloud Bookstore ${ }^{4}$, una plataforma para la distribución y venta online de contenidos digitales. Este producto está orientado a organizaciones en los sectores educativo, editorial y cultural. Esta plataforma permite a cualquier organización ofrecer, vender y distribuir contenidos digitales a través de un canal propio, sin depender de intermediarios (y de los costes que esa dependencia implica). Uno de los objetivos en el diseño y desarrollo de este producto fue identificar y dar soporte a los requisitos que cualquier cliente potencial pudiera necesitar. Sin embargo, es posible que en muchos casos sean necesarias ciertas adaptaciones del producto a las necesidades particulares de un cliente, por lo que la mantenibilidad del producto cobra una gran importancia de cara a su comercialización.

Las expectativas de Enxenio de cara al proyecto de evaluación y certificación eran asegurar que el producto presentara un nivel de mantenibilidad alto (por la importancia que la mantenibilidad tiene de cara a la evolución y adaptación del producto a los nuevos clientes), y que este nivel de mantenibilidad estuviera certificado por un organismo independiente y ampliamente reconocido.

\section{B. Proceso de evaluación y certificación de la mantenibilidad}

La evaluación y certificación del producto se llevó a cabo en tres fases. El laboratorio AQC Lab realizó una primera evaluación del producto en base al modelo de calidad presentado en secciones anteriores. Esta primera evaluación del producto dio lugar a un informe detallado con el nivel de calidad alcanzado en cada una de las características que forman el modelo, y con un informe exhaustivo de aquellos componentes del código fuente del producto que incumplen alguna de las reglas y métricas de mantenibilidad

El nivel global de mantenibilidad alcanzado por el producto inicialmente fue un nivel 2, lo que está por debajo del umbral mínimo para poder optar al certificado de AENOR (nivel 3 o superior).

Esta primera evaluación permitió a Enxenio conocer con mucho detalle (nivel de línea de código) aquellos aspectos del producto que podrían suponer un problema para su mantenibilidad en el futuro, como eran:

- Baja Analizabilidad: lo que penalizaba al equipo de desarrollo a la hora de poder detecta qué partes del producto implementaban una funcionalidad concreta.

- Baja Modularidad: lo que suponía un riesgo a la hora de corregir un módulo sin que otros independientes se vieran afectados.

- Baja Capacidad para ser modificado: lo que afectaba a la facilidad para introducir cambios en el producto sin introducir nuevos errores.

- Baja Capacidad para ser reutilizado: lo que representaba un riesgo de que los módulos del producto no se pudieran reutilizar por su acoplamiento con otras partes.

- Baja Capacidad para ser probado: lo que impedía confiar en que las pruebas realizadas al sistema fueran lo suficientemente completas.

En base al informe proporcionado por AQC se llevó a cabo un proyecto de mejora con el fin de corregir aquellos aspectos señalados en el informe de evaluación.

Tras la refactorización y mejora del producto por parte de Enxenio, AQC llevó a cabo una nueva evaluación del producto, en la que el nivel de mantenibilidad alcanzado fue 4. De nuevo, se emitió un informe con los resultados de la evaluación, detallando el nivel alcanzado para cada una de las características que contempla el modelo de mantenibilidad. Los resultados de ambas evaluaciones se muestran en la Fig. 5.

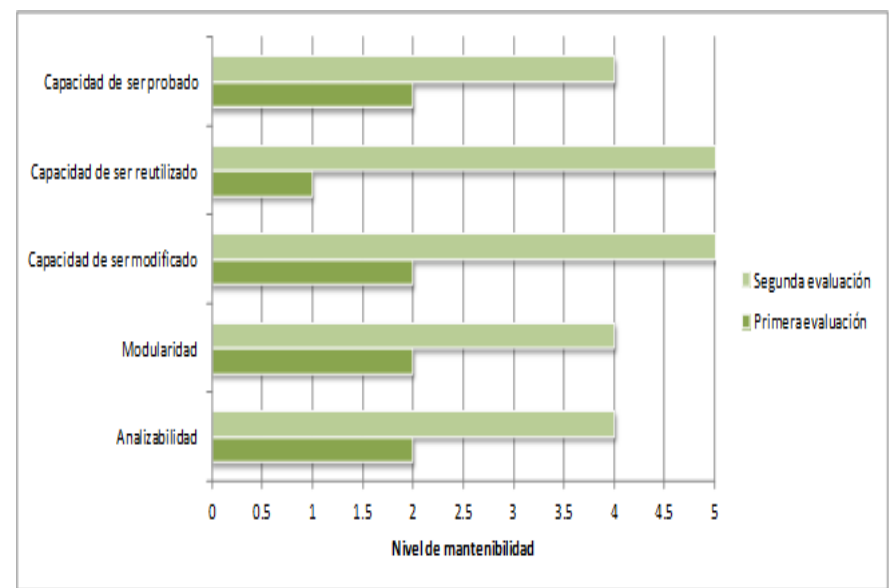

Fig. 5. Ciclo de Evaluación y Certificación del Producto Software 
Como se puede ver en la figura, el nivel de mantenibilidad se mejoró en todas las sub-características de calidad, especialmente en los casos de capacidad de ser reutilizado y capacidad de ser modificado. El resultado de las dos evaluaciones y las acciones de refactorización suponen una mejora efectiva del nivel de mantenibilidad del producto, lo que redundará en menores costes de mantenimiento en un futuro. El proyecto de mejora de la mantenibilidad del producto supuso para Enxenio un esfuerzo de 80 horas, mientras que las dos evaluaciones realizadas supusieron un esfuerzo total de 70 horas para los técnicos del laboratorio AQC Lab.

Tras los resultados de la segunda evaluación, Enxenio solicitó la certificación del nivel de mantenibilidad del producto a AENOR. Tras analizar el informe emitido por AQC Lab, AENOR llevó a cabo una auditoría in-situ de la empresa durante una jornada de trabajo. En dicha auditoría se comprobó que la empresa cuenta con los recursos e infraestructura necesarios para garantizar la mantenibilidad del producto en el futuro, obteniendo como resultado el certificado de calidad de producto emitido por AENOR.

Los principales resultados obtenidos por parte de Enxenio son:

- Alto nivel de mantenibilidad del producto: la primera consecuencia obtenida por Enxenio en el proyecto piloto es que la mantenibilidad del producto se ha mejorado de forma efectiva. Esta mejora redundará en menores costes de mantenimiento, evolución y adaptación del producto a las necesidades de los nuevos clientes. Desde este punto de vista, el esfuerzo de refactorización del producto se ve como una inversión que supondrá un ahorro de costes en el futuro.

- Modelo de referencia independiente y accesible: aunque desde hace tiempo existen distintas herramientas de análisis del código fuente y extracción de métricas, el modelo desarrollado por AQC y AENOR proporciona un marco de referencia independiente contra el que medir las características de cualquier producto software.

- Certificado de calidad emitido por entidades acreditadas y reconocidas: para Enxenio, el hecho de que el producto cuente con un certificado de calidad respaldado por entidades como AENOR y AQC supone un sello de garantía de gran importancia para los potenciales clientes. En la Fig. 6 se muestra el certificado conseguido por Enxenio con su producto software.

- Aplicación en futuros proyectos como garantía para el cliente: contar con un sello de calidad de producto (y no solo de proceso) emitido por una entidad independiente es una de las mejores garantías de calidad que se pueden ofrecer a un cliente, ya sea en la adaptación de un producto estándar o en el desarrollo de un producto a medida.

- Así, para Enxenio la evaluación y certificación de calidad de producto conforme a ISO 25000 supone una forma de ofrecer a sus futuros clientes una garantía de calidad aún mayor.

- Incorporación de evaluación de producto a los procesos de la empresa: tras esta experiencia, Enxenio ha incorporado a sus procesos de gestión y desarrollo aspectos de evaluación de la calidad de los productos. Así, la gestión de la calidad no se centra solo en los procesos, sino que llega a los productos generados.

\section{CONCLUSIONES Y TRABAJO FUTURO}

La calidad del producto software es una de las principales preocupaciones tanto para las empresas desarrolladoras, como para los organismos que lo adquieren. El presente artículo ha presentado una solución, formada por un ecosistema completo, que permiten realizar la evaluación del producto software y alcanzar posteriormente una certificación de calidad. Además, para mostrar la aplicación práctica de la evaluación y certificación de la calidad del producto software, se han presentado también los detalles de un proyecto piloto de evaluación y certificación realizado, con los datos concretos obtenidos por una de las empresas participantes en dicho proyecto.

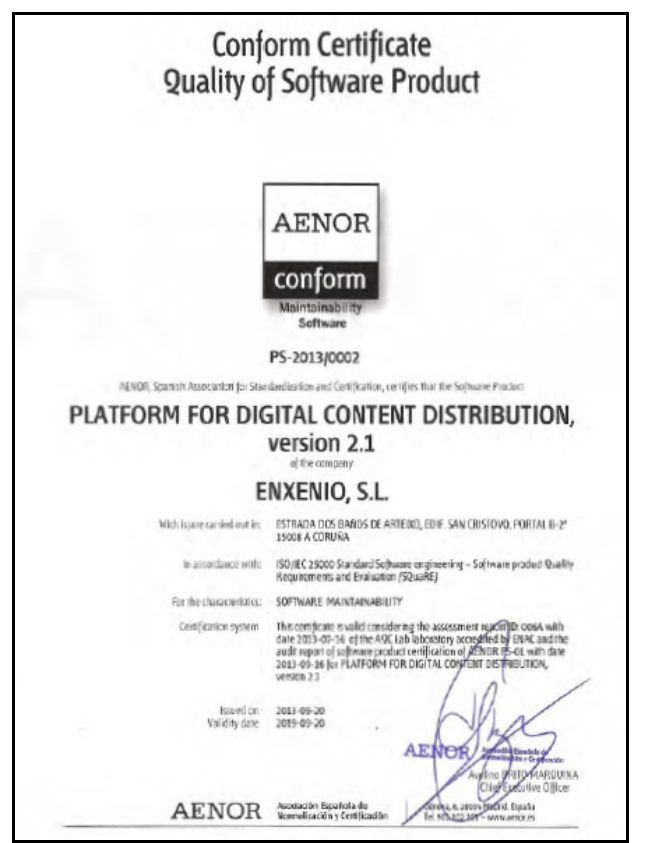

Fig. 6. Ciclo de Evaluación y Certificación del Producto Software

Entre los beneficios obtenidos, las empresas participantes destacaron haber reducido hasta en un $75 \%$ las incidencias correctivas, hasta en un $40 \%$ el tamaño de sus productos y hasta en un $30 \%$ los tiempos en las tareas de mantenimiento. Y tras este piloto, son varios los productos que a nivel nacional e internacional ya se han evaluado siguiendo este nuevo esquema, estando algunos de ellos ya en fase de certificación.

Por otro lado, indicar que AENOR y AQC Lab siguen trabajando para ampliar el alcance de las evaluaciones y certificaciones del producto software, de manera que se puedan abordar poco a poco todas las características de calidad identificadas por la familia ISO/IEC 25000.

\section{AGRADECIMIENTOS}

Esta investigación forma parte de los proyectos: GEODASBC (Ministerio de Economía y Competitividad, TIN201237493-C03-01), SDGear (Ministerio de Industria, Energía y Turismo, TSI-100104-2014-4) e IMPACTUM (Consejería de Educación, Ciencia y Cultura de la JCCM, PEII-2014-017-A), todos ellos dentro del Fondo Europeo de Desarrollo Regional FEDER.

\section{REFERENCIAS}

[1] M.G. Piattini and J. Garzás, Fábricas de software: experiencias, tecnologías y organización ( ${ }^{a}$ edición actualizada). 2010, Paracuellos del Jarama (Madrid): RA-MA. 
[2] Standish-Group, The CHAOS Manifesto: Think Big, Act Small. 2013, The Standish Group.

[3] B.W. Boehm, et al., Characteristics of Software Quality. 1978: North-Holland.

[4] ISO, ISO/IEC 9126, Software Product Evaluation-Quality Characteristics and Guidelines for their Use. 2001, International Organization for Standardization.

[5] ISO, ISO/IEC 14598-5:1998 - Information technology -Software product evaluation -- Part 5: Process for evaluators. 1998, International Organization for Standardization: Ginebra.

[6] I. Heitlager, T. Kuipers and J. Visser, A Practical Model for Measuring Maintainability, in Quality of Information and Communications Technology, 2007. QUATIC 2007. 2007. p. 3039.

[7] ISO, ISO/IEC 25000, Systems and software engineering -Systems and software Quality Requirements and Evaluation (SQuaRE) -- Guide to SQuaRE. 2014: Ginebra, Suiza.

[8] M. Rodríguez and M. Piattini, Systematic review of software product certification, in CISTI 2012: 7th Iberian Conference on Information Systems and Technologies. 2012: Madrid. p. 631636.

[9] B. Kitchenham, Guideline for performing Systematic Literature Reviews in Software Engineering. Version 2.3. 2007, University of Keele (Software Engineering Group, School of Computer Science and Mathematics) and Durham (Department of Computer Science).

[10] J. Biolchini, P. Gomes, A. Cruz and G. Travassos, Systematic Review in Software Engineering. 2005, Systems Engineering and Computer Science Department, UFRJ: Rio de Janeiro, Brazil.

[11] P. Heck, M. Klabbers and M. van Eekelen, A software product certification model. Software Quality Journal, 2009. 18(1): p. 37-55.

[12] F. Carvalho, S.R.L. Meira, B. Freitas and J. Eulino, Embedded software component quality and certification. Conference Proceedings of the EUROMICRO, 2009: p. 420-427.

[13] E. Burger and R. Reussner, Performance certification of software components. Electronic Notes in Theoretical Computer Science, 2011. 279(2): p. 33-41.

[14] A. Serebrenik, A. Mishra, T. Delissen and M. Klabbers, Requirements certification for offshoring using LSPCM. Proceedings - 7th International Conference on the Quality of Information and Communications Technology, QUATIC 2010, 2010: p. 177-182.

[15] J.H. Yahaya, A. Deraman and A.R. Hamdan, SCfM_PROD: A software product certification model. 2008 3rd International Conference on Information and Communication Technologies: From Theory to Applications, ICTTA, 2008.

[16] J. Yahaya, A. Deraman and A.R. Hamdan, Continuously ensuring quality through software product certification: A case study. 2010 International Conference on Information Society, iSociety 2010, 2010: p. 183-188.

[17] J. Hatcliff, et al., A Software Certification Consortium and its Top 9 Hurdles. Electronic Notes in Theoretical Computer Science, 2009. 238(4): p. 11-17.

[18] J. Morris, G. Lee, K. Parker, G.A. Bundell and C.P. Lam, Software component certification. Computer, 2001. 34(9): p. 3036.

[19] R. Baggen, J.P. Correia, K. Schill and J. Visser, Standardized code quality benchmarking for improving software maintainability. Software Quality Journal, 2012. 20(2): p. 287307

[20] A. Alvaro, E.S. De Almeida and S.L. Meira, Towards a software component certification framework. Proceedings - International Conference on Quality Software, 2007: p. 298-303.

[21] C.M. Fernández and M. Piattini, Modelo para el Gobierno de las TIC basado en normas ISO. $2^{a}$ ed. 2015, Madrid: AENOR.
[22] M. Rodríguez, C.M. Fernández and M. Piattini, ISO/IEC 25000 Calidad del Producto Software. AENOR. Revista de la Normalización y la Certificación, 2013(288): p. 30-35.

[23] ISO, ISO/IEC 25010, Systems and software engineering Systems and software Quality Requirements and Evaluation (SQuaRE) - System and software quality models. 2011: Ginebra, Suiza.

[24] ISO, ISO/IEC 25040 Systems and software engineering Systems and software Quality Requirements and Evaluation (SQuaRE) - Evaluation process. 2011: Ginebra, Suiza.

[25] P. Tomas, M.J. Escalona and M. Mejias, Open source tools for measuring the Internal Quality of Java software products. A survey. Computer Standards \& Interfaces, 2013(36): p. 244-255.

[26] J. Verdugo, M. Rodríguez and M. Piattinni, Using Agile Methods to Implement a Laboratory for Software Product Quality Evaluation, in 15th International Conference on Agile Software Development (XP 2014). 2014: Roma (Italia).

[27] ISO, ISO/IEC 17025:2005. General requirements for the competence of testing and calibration laboratories. 2005, International Organization for Standardization.

[28] M. Rodríguez and M. Piattini, Entorno para la Evaluación y Certificación de la Calidad del Producto Software, in XIX Jornadas de Ingeniería del Software y Bases de Datos JISBD’2014. 2014: Cadiz. p. 163-176.

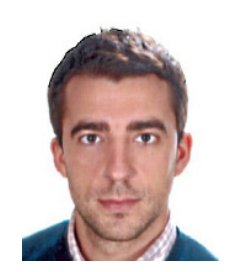

Moisés Rodríguez Monje. Ingeniero Superior en Informática y Máster en Tecnologías Informáticas Avanzadas por la Universidad de Castilla-La Mancha. Auditor CISA por ISACA y auditor Jefe por AENOR en ISO/IEC 15504 - 12207. SocioDirector en Alarcos Quality Center y Director General del laboratorio AQC Lab para la evaluación de la calidad del producto software bajo ISO/IEC 25000

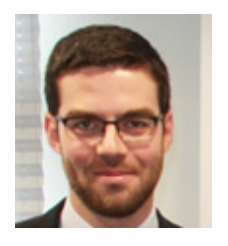

Óscar Pedreira Fernández. Doctor e Ingeniero en Informática por la Universidad de A Coruña. Profesor en la Universidad de A Coruña y Socio de la empresa Enxenio, spin-off del Laboratorio de Bases de datos. Responsable de los sistemas de gestión de calidad ISO 27001, ISO 15504 e ISO 25000 .

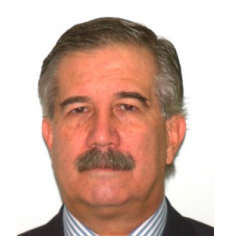

Carlos Manuel Fernández. Ingeniero en Informática por la Universidad Politécnica de Madrid y Máster en Dirección de Empresas por el CECO-Madrid. Auditor CISA y CISM por ISACA. Actualmente es Profesor en Máster de TICs (entre otras universidades: UPM, UAH, UNIR, URJC, etc.). En la actualidad es Gerente de Certificaciones TICs en AENOR, coordinando más de 500 empresas en España, LATAM y Europa. 